\title{
Infective endocarditis with Lactococcus garvieae in Japan: a case report
}

\author{
Yukiko Watanabe ${ }^{1,2}$, Toshio Naito ${ }^{1,2^{*}}$, Ken Kikuchi ${ }^{1,2}$, Yu Amari ${ }^{1}$, Yuki Uehara ${ }^{1,2}$, Hiroshi Isonuma ${ }^{1}$, Teruhiko Hisaoka ${ }^{1}$, \\ Terutoyo Yoshida ${ }^{4}$, Kenji Yaginuma ${ }^{3}$, Norihide Takaya ${ }^{3}$, Hiroyuki Daida ${ }^{3}$ and Keiichi Hiramatsu ${ }^{2}$
}

\begin{abstract}
Introduction: Lactococcus garvieae is a well-recognized fish pathogen, and it is considered a rare pathogen with low virulence in human infection. We describe the 11th case of L. garvieae infective endocarditis reported in the literature, and the first reported case in Japan.
\end{abstract}

Case presentation: We report a case of a 55-year-old Japanese woman who had native valve endocarditis with $L$. garvieae. The case was complicated by renal infarction, cerebral infarction, and mycotic aneurysms. After antimicrobial treatment, she was discharged from the hospital and is now well while being monitored in the outpatient clinic.

Conclusion: We encountered a case of L. garvieae endocarditis that occurred in a native valve of a healthy woman. The $16 \mathrm{~S}$ ribosomal RNA gene sequencing was useful for the identification of this pathogen. Although infective endocarditis with L. garvieae is uncommon, it is possible to treat high virulence clinically.

\section{Introduction}

Lactococcus garvieae was first isolated from cases of bovine mastitis [1], and it is rarely reported in human infection. The first human infection was documented as infective endocarditis (IE) in 1991 [2]. Since then, it has been identified as a pathogen in IE [3-9], liver abscess [10], and osteomyelitis [4]. We describe the 11th case of L. garvieae IE reported in the literature, and the first reported case in Japan.

\section{Case presentation}

A 55-year-old healthy Japanese woman presented to our hospital with a two-month history of malaise and myalgia, and she had had a fever for one month. The patient had no significant medical history and no relevant history regarding pets, travel, dental treatment, or drug abuse. Her occupational history was that she worked at an import grocery store. During her physical examination, we noted her body temperature of $37.6^{\circ} \mathrm{C}$, a grade 2 systolic murmur at the apex, and a painful $1 \mathrm{~mm}$ black induration on her right forefinger. Trans-thoracic

\footnotetext{
* Correspondence: naito@juntendo.ac.jp

'Department of General Medicine, Juntendo University School of Medicine,

2-1-1 Bunkyo-ku, Hongo, Tokyo 113-8421, Japan

Full list of author information is available at the end of the article
}

echocardiography showed a $1 \mathrm{~cm}$ mobile mass on the mitral valve with moderate mitral regurgitation. Her laboratory tests showed a white blood cell count of $8200 / \mu \mathrm{L}$, a C-reactive protein level of $6.6 \mathrm{mg} / \mathrm{dL}$, and a hemoglobin level of $9.9 \mathrm{~g} / \mathrm{dL}$. Gram-positive cocci forming pairs or short chains were shown by Gram staining of three sets of blood cultures drawn at the time of admission.

IE was diagnosed on the basis of the Duke criteria for the diagnosis of infective endocarditis [11] (two major and two minor criteria) and antibiotic treatment with benzylpenicillin 3 million units every four hours and gentamicin $60 \mathrm{mg}$ every eight hours was started, as Streptococcus sp. was suspected as the causative pathogen. Subsequently, the rapid ID32 Strep (bioMérieux, Marcy-l'Etoile, France) profile (code 34323501010) revealed $L$. garvieae. We also performed molecular species identification by $16 \mathrm{~S}$ ribosomal RNA gene (16S rRNA) sequencing, and the almost full-length $16 \mathrm{~S}$ rDNA matched L. garvieae (99.9\%). The results of an anti-microbial susceptibility test using Etest strips (AB Biodisk, Dalvagen, Solna, Sweden) showed the following values: erythromycin $0.25 \mathrm{mg} / \mathrm{L}$, clindamycin $\geq 256 \mathrm{mg} /$ $\mathrm{L}$, vancomycin $0.38 \mathrm{mg} / \mathrm{L}$, linezolid $2.0 \mathrm{mg} / \mathrm{L}$, penicillin $0.5 \mathrm{mg} / \mathrm{L}$, ceftriaxone $0.38 \mathrm{mg} / \mathrm{L}$, gentamicin $1.5 \mathrm{mg} / \mathrm{L}$, 
and streptomycin $64 \mathrm{mg} / \mathrm{L}$. Considering these results, we changed the anti-microbial treatment to intravenous ceftriaxone 2 g every 12 hours and gentamicin $80 \mathrm{mg}$ every eight hours intraveneously.

Meanwhile, her body temperature rose to $39^{\circ} \mathrm{C}$ with left lower back pain on her sixth day in the hospital. Left-sided renal infarction was diagnosed on the basis of contrast-enhanced computed tomography (CT) (Figure $1)$. On the 12th hospital day, cerebral infarction occurred, followed by a sudden onset of right-sided hemiparesis and slurred speech (National Institutes of Health Stroke Scale (NIHSS) score 12). Diffusionweighted MRI showed a cerebral infarction of the middle cerebral artery area (Figure 2). Furthermore, cerebral mycotic aneurysms were detected by three-dimensional CT angiography on the 24th hospital day (Figure 3 ). The patient also developed aspiration pneumonia after the cerebral infarction complication.

Anti-microbial treatment was completed in nine weeks, including the treatment for pneumonia. Several repeat blood cultures thereafter were all negative. The vegetation on the mitral valve was no longer detected by trans-esophageal echocardiography performed just before she was discharged from the hospital. We found by $\mathrm{CT}$ that her left-sided renal infarction had improved, and the consequences of her cerebral infarction had also improved with only right-sided muscular weakness (NIHSS score 2). She was discharged from the hospital, and she is now well while being monitored in the outpatient clinic.

\section{Discussion}

Genus Lactococcus was separated from genus Streptococcus in 1985 based on DNA-DNA relatedness and 16S rRNA sequencing data published by Ludwig et al. [12]. The genus Lactococcus was classified into eight species

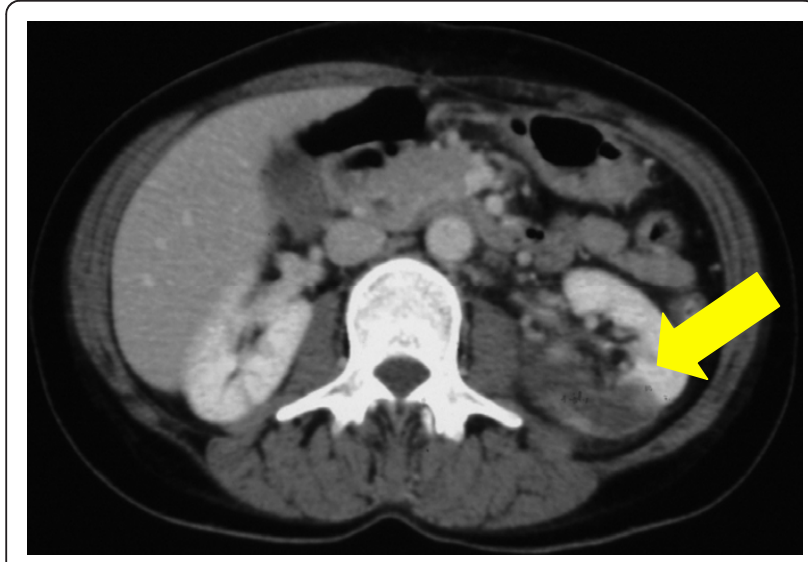

Figure 1 Contrast-enhanced abdominal computed tomography Yellow arrow indicates left renal infarction.

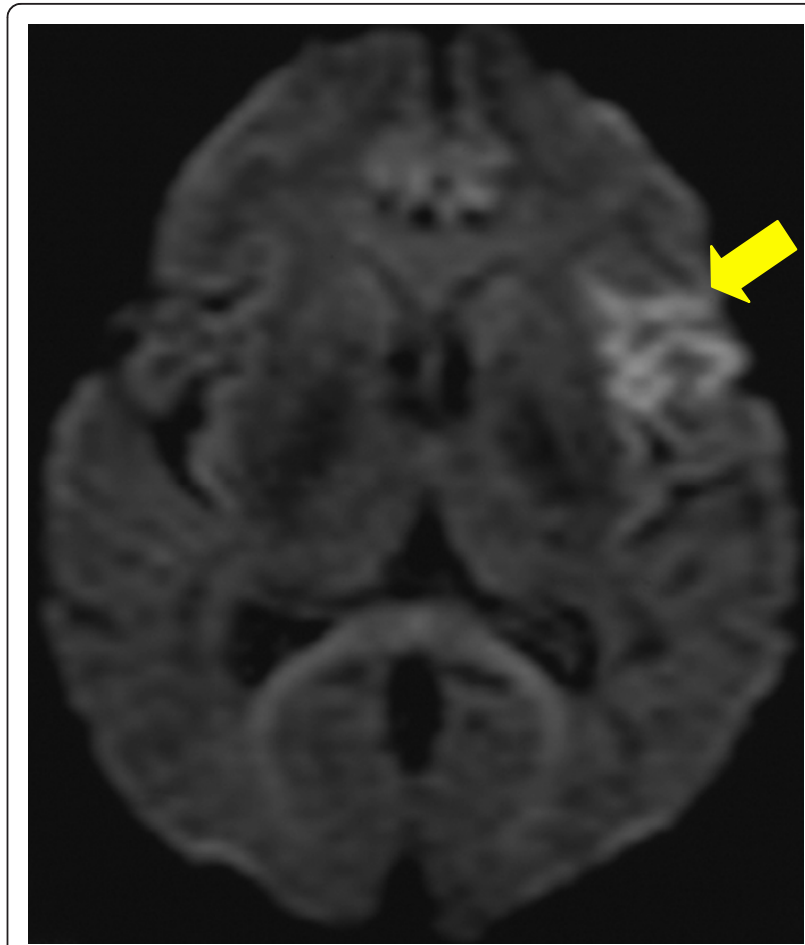

Figure 2 Diffusion-weighted MRI scan. Yellow arrow indicates the middle cerebral artery areas of high signal intensity.

and sub-species by Facklam and Elliott in 1995 [13] and $\mathrm{Pu}$ et al. in 2002 [14]: L. lactis ssp. lactis, L. lactis ssp. cremoris, L. lactis ssp. hordniae, L. garvieae, L. piscium, L. plantarum, L. raffinolactis, and L. xyloses. Cases involving the $L$. garvieae pathogen in human infection are rarely reported [2-10]. The major clinical presentation is IE, which has been reported in 10 cases in the

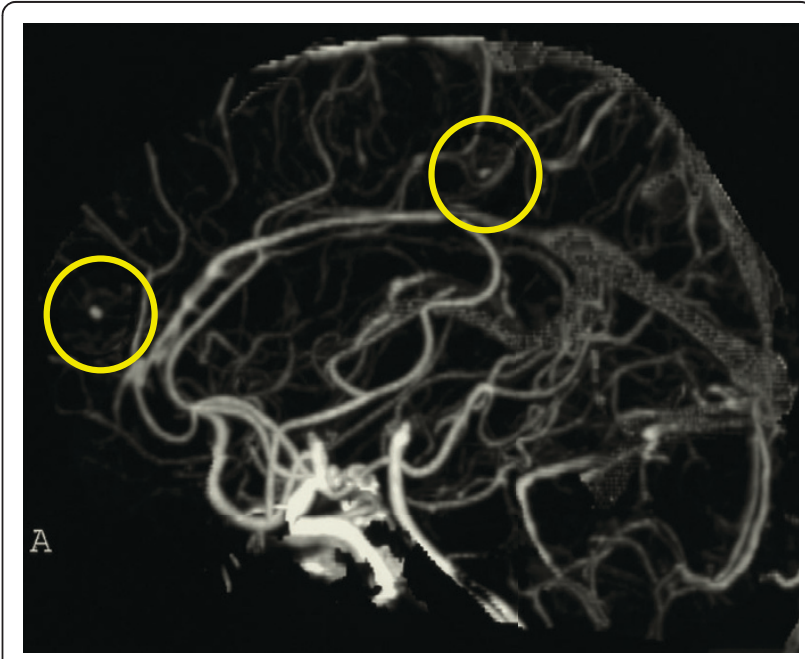

Figure 3 Three-dimensional computed tomography angiography. The two yellow circles indicate cerebral mycotic aneurysms. 
literature [2-9]. Five of ten cases involved native valves $[3,6-9]$, and four cases required surgery for valve replacement $[3,6,8,9]$. In addition, two cases were complicated by cerebral infarction or hemorrhage $[3,9]$. $L$. garvieae IE is uncommon; however, the virulence of $L$. garvieae might be underestimated. The treatment for $L$. garvieae IE is not standardized, because the exact criterion of the susceptibility test has not been established. In the published cases, penicillin combined with gentamicin, amoxicillin combined with netilmicin, ampicillin, ceftriaxone, and vancomycin were used as anti-microbial treatment. In our case, the results of the anti-microbial susceptibility test of the isolate using the Etest method was susceptibility to ceftriaxone $(0.38 \mathrm{mg} / \mathrm{L})$, erythromycin $(0.25 \mathrm{mg} / \mathrm{L})$, vancomycin $(0.38 \mathrm{mg} / \mathrm{L})$, and linezolid ( $2 \mathrm{mg} / \mathrm{L})$, less susceptibility to penicillin $(0.5 \mathrm{mg} / \mathrm{L})$, and resistance to clindamycin (> $256 \mathrm{mg} / \mathrm{L}$ ).

Elliott and Facklam [15] reported that clindamycin resistance is a feature of L. garvieae, and this feature can be used to distinguish it from other lactococci. To confirm the precise identification, various molecular techniques are available: whole-cell protein analysis, $16 \mathrm{~S}$ rRNA gene sequencing, and $\operatorname{sod} A_{\text {int }}$ (an internal fragment of the $\operatorname{sod} A$ gene encoding the manganese-dependent superoxide dismutase) gene sequencing.

L. garvieae has pathogenicity for several fish species, ruminants, and humans. Therefore, it could be a potentially zoonotic infection. Investigators in one recent report hypothesized that eating infected raw fish carrying L. garvieae caused its infection [7]. To investigate the relationship between our isolate and fish isolates, the immunological property was examined by using several anti-sera against $L$. garvieae that have previously been produced by fish in Japan. The results showed that our isolate did not have a capsule detected by any anti-capsule antibody for fish isolates. Pulsed-field gel electrophoresis also showed that the pattern of our isolate was quite different from that of fish-derived strains (data not shown). Vela et al. [16] found great phenotypic heterogeneity and genetic diversity among the $L$. garvieae isolates from fish, cows, water buffalo, and humans, with a generally good correlation between the phenotypic and genetic properties of $L$. garvieae. As the origin of the L. garvieae strain in our patient could not be clarified by the investigations used to detect Japanese fish pathogens, other causes of her infection, such as cow's milk or cheeses, are possible. To confirm the etiology of the transmission of L. garvieae, larger molecular epidemiological studies including investigating causes from the environment, animals, and humans must be carried out.

\section{Conclusion}

Herein we report the first case of $L$. garvieae IE in Japan. Molecular biological investigation techniques such as 16S ribosomal RNA gene sequencing are useful for the identification of L. garvieae. Although L. garvieae is considered a rare pathogen with low virulence in human infection, it could cause native valve endocarditis in healthy people. It might be necessary to review the precise estimation of the pathogenicity of L. garvieae.

\section{Consent}

Written informed consent was obtained from the patient for publication of this case report and any accompanying images. A copy of the written consent is available for review by the Editor-in-Chief of this journal.

\section{Acknowledgements}

We thank Dr Shigeki Misawa, Clinical Laboratory, Juntendo University Hospital, Tokyo, Japan, for phenotypic characterization of the bacterial isolate.

\section{Author details}

'Department of General Medicine, Juntendo University School of Medicine, 2-1-1 Bunkyo-ku, Hongo, Tokyo 113-8421, Japan. ²Department of Infection Control Science, Juntendo University School of Medicine, 2-1-1 Bunkyo-ku, Hongo, Tokyo 113-8421, Japan. ${ }^{3}$ Department of Cardiology, Juntendo University School of Medicine, 2-1-1 Bunkyo-ku, Hongo, Tokyo 113-8421, Japan. ${ }^{4}$ Department of Fisheries, Faculty of Agriculture, Miyazaki University, Miyazaki City, Miyazaki 889-2192, Japan.

\section{Authors' contributions}

YW drafted and edited the manuscript. TN, KK, YA, YU, HI, TH, and KH helped draft the manuscript. KY, NT, and HD helped in the patient's treatment after the diagnosis was made. TY helped in the investigation of $L$. garvieae as a fish pathogen. All authors read and approved the final manuscript.

\section{Competing interests}

The authors declare that they have no competing interests.

Received: 13 December 2010 Accepted: 9 August 2011

Published: 9 August 2011

\section{References}

1. Collins MD, Farrow JAE, Phillips BA, Kandler O: Streptococcus garvieae sp. nov. and Streptococcus plantarum sp. nov. J Gen Microbiol 1983, 129:3427-3431

2. Furutan NP, Breiman RF, Fischer MA, Facklam RR: Lactococcus garvieae infections in humans: a cause of prosthetic valve endocarditis [abstract C297]. Proceedings of the 91st General Meeting of the American Society for Microbiology Dallas: American Society of Microbiology; 1991, 109.

3. Fefer JJ, Ratzan KR, Sharp SE, Saiz E: Lactococcus garvieae endocarditis: report of a case and review of the literature. Diagn Microbiol Infect Dis 1998, 32:127-130.

4. James PR, Hardman SM, Patterson DL: Osteomyelitis and possible endocarditis secondary to Lactococcus garvieae: a first case report. Postgrad Med J 2000, 76:301-303.

5. Fihman V, Raskine L, Barrou Z, Kiffel C, Riahi J, Berçot B, Sanson-Le Pors MJ: Lactococcus garvieae endocarditis: identification by $16 \mathrm{~S}$ rRNA and sodA sequence analysis. J Infect 2006, 52:e3-e6.

6. Vinh DC, Nichol KA, Rand F, Embil JM: Native-valve bacterial endocarditis caused by Lactococcus garvieae. Diagn Microbiol Infect Dis 2006, 56:91-94.

7. Wang CY, Shie HS, Chen SC, Huang JP, Hsieh IC, Wen MS, Lin FC, Wu D: Lactococcus garvieae infections in humans: possible association with aquaculture outbreaks. Int J Clin Pract 2007, 61:68-73.

8. Yiu KH, Siu CW, To KK, Jim MH, Lee KL, Lau CP, Tse HF: A rare cause of infective endocarditis: Lactococcus garvieae. Int J Cardiol 2007, 114:286-287. 
9. Li WK, Chen YS, Wann SR, Liu YC, Tsai HC: Lactococcus garvieae endocarditis with initial presentation of acute cerebral infarction in a healthy immunocompetent man. Intern Med 2008, 47:1143-1146.

10. Mofredj A, Baraka D, Kloeti G, Dumont JL: Lactococcus garvieae septicemia with liver abscess in an immunosuppressed patient. Am J Med 2000, 109:513-514

11. Durack D, Lukes A, Bright D, Duke Endocarditis Service: New criteria for diagnosis of infective endocarditis: utilization of specific echocardiographic findings. Am J Med 1994, 96:200-209.

12. Ludwig W, Seewaldt E, Kilpper-Bälz R, Schleifer KH, Magrum L, Woese CR, Fox GE, Stackebrandt E: The phylogenetic position of Streptococcus and Enterococcus. J Gen Microbiol 1985, 131:543-551.

13. Facklam R, Elliott JA: Identification, classification, and clinical relevance of catalase-negative, gram-positive cocci, excluding the streptococci and enterococci. Clin Microbiol Rev 1995, 8:479-495.

14. Pu ZY, Dobos M, Limsowtin GK, Powell IB: Integrated polymerase chain reaction-based procedures for the detection and identification of species and subspecies of the Gram-positive bacterial genus Lactococcus. J Appl Microbiol 2002, 93:353-361.

15. Elliott JA, Facklam RR: Antimicrobial susceptibilities of Lactococcus lactis and Lactococcus garvieae and a proposed method to discriminate between them. J Clin Microbiol 1996, 34:1296-1298.

16. Vela Al, Vázquez J, Gibello A, Blanco MM, Moreno MA, Liébana P, Albendea C, Alcalá B, Mendez A, Domínguez L, Fernández-Garayzábal JF: Phenotypic and genetic characterization of Lactococcus garvieae isolated in Spain from lactococcosis outbreaks and comparison with isolates of other countries and sources. J Clin Microbiol 2000, 38:3791-3795.

doi:10.1186/1752-1947-5-356

Cite this article as: Watanabe et al.: Infective endocarditis with

Lactococcus garvieae in Japan: a case report. Journal of Medical Case Reports 2011 5:356.

\section{Submit your next manuscript to BioMed Central and take full advantage of:}

- Convenient online submission

- Thorough peer review

- No space constraints or color figure charges

- Immediate publication on acceptance

- Inclusion in PubMed, CAS, Scopus and Google Scholar

- Research which is freely available for redistribution

Submit your manuscript at www.biomedcentral.com/submit 